\title{
Hierarchical Newton Iterative Parameter Estimation of a Class of Input Nonlinear Systems Based on the Key Term Separation Principle
}

\author{
Cheng Wang $\mathbb{D}^{1},{ }^{1}$ Kaicheng $\mathrm{Li}^{2}{ }^{2}$ and Shuai $\mathrm{Su}^{2}$ \\ ${ }^{1}$ Key Laboratory of Advanced Process Control for Light Industry (Ministry of Education), Jiangnan University, Wuxi 214122, China \\ ${ }^{2}$ National Engineering Research Center of Rail Transportation Operation and Control System, Beijing Jiaotong University, \\ Beijing 100044, China \\ Correspondence should be addressed to Cheng Wang; artiefly@gmail.com
}

Received 23 May 2018; Revised 27 July 2018; Accepted 7 August 2018; Published 24 October 2018

Academic Editor: Jing $\mathrm{Na}$

Copyright (c) 2018 Cheng Wang et al. This is an open access article distributed under the Creative Commons Attribution License, which permits unrestricted use, distribution, and reproduction in any medium, provided the original work is properly cited.

\begin{abstract}
This paper investigates the identification problem for a class of input nonlinear systems whose disturbance is in the form of the moving average model. In order to improve the computation complexity, the key term separation principle is introduced to avoid the redundant parameter estimation. Based on the decomposition technique, a hierarchical Newton iterative identification method combining the key term separation principle is proposed for enhancing the estimation accuracy and handling the computational load with the presence of the high dimensional matrices. In the identification procedure, the unknown internal items or vectors are replaced with their iterative estimates. The effectiveness of the proposed identification methods is shown via a numerical simulation example.
\end{abstract}

\section{Introduction}

In the modern cyber-physical system, including robotics systems $[1,2]$, railway control systems $[3,4]$, etc., system identification plays an important role in establishing relationship between the virtual system and the real world by using the modeling technique $[5,6]$. Generally, modeling techniques can be split into two groups: nonparametric modeling and parametric modeling. Nonparametric modeling, so-called black or grey box modeling, ignores the mechanism of the system and instead concentrates on studying the relationship between the system input and output $[7,8]$. In contrast, parametric modeling focuses on estimating the parameters where the model structure is fixed on the basis of the first principle or others $[9,10]$.

The parametric modeling or parameter estimation relies deeply on adaptive algorithm [11, 12]. The core idea of adaptive parameter estimation is to recursively adjust the parameters by using the residuals, which makes the estimates approximately approach the true value. Under this framework of adaptation, the recursive least squares algorithms [13, 14], the stochastic gradient algorithms [15], and the iterative algorithms $[16,17]$ are well developed and underpin several heuristic or bioinspired learning algorithms [18-20]. For example, we use the adaptive algorithms such as gradient descent algorithm for learning the weights in the neural networks or training the fitness in the genetic algorithms.

Under the adaptive identification framework, the Newton iterative algorithm can produce high accuracy estimation with fast convergence property [21-23]. However, the identification process involves heavy matrix computation. In the industry field, the wireless embedded devices are so vulnerable to the complexity of the algorithm that high power-consumed computation needs be avoided. The decomposition technique, as an effective tool to improve the computation efficiency, is applied into many identification algorithms. For example, Ding et al. decomposed Hammerstein-controlled autoregressive systems into three subsystems and employed the auxiliary model identification 
idea for handling unknown parameters coupled in each subsystem [24]. Ma et al. proposed a decomposition-based recursive least squares identification methods for multivariate pseudolinear systems using the multi-innovation theory [25].

Block-oriented identification of the nonlinear systems such as the Hammerstein models [26, 27], the Wiener models [28], or the Hammerstein-Wiener models has become the active topic in the research of nonlinear parametric modeling, and many identification methods have been developed [29-31]. Liu and Bai presented an iterative identification algorithm for Hammerstein systems and studied its convergence [32]. The iterative algorithms utilize the batch of data for parameter estimation and are used in various off-line applications. Ding et al. focused on on-line identification and proposed a recursive least squares parameter estimation algorithm for output nonlinear autoregressive systems [33]. Recently, Ma et al. used the variational Bayesian approach to identify the Hammerstein parameter varying systems for chemical dynamic processes, which often work under various conditions bringing the varying model parameters [34].

The general Hammerstein model or input nonlinear model presented by a memoryless nonlinear block and a linear dynamic subsystem can be formulated.

$$
y(t)=G(z) \bar{u}(t)+N(z) v(t)
$$

where $y(t) \in \mathbb{R}$ and $u(t) \in \mathbb{R}$ are the measured system output and input, $v(t) \in \mathbb{R}$ is the stochastic white noise, $G(z)$ and $N(z)$ are the transfer functions of the system model and the noise model, the intermediate variable $x(t)=G$ $(z) \bar{u}(t)$ denotes the noise-free system output, and $w(t)=$ $N(z) v(t)$ denotes the noise model output. The unmeasurable variable $\bar{u}(t)$ is the output of the nonlinear block and can be represented as the linear combination of the known parameter vectors $\left(\gamma_{1}, \gamma_{2}, \cdots, \gamma_{m}\right)$ and the known basis $f=\left(f_{1}, f_{2}, \cdots, f_{m}\right)$ :

$$
\begin{aligned}
\bar{u}(t) & =\gamma_{1} f_{1}(u(t))+\gamma_{2} f_{2}(u(t))+\cdots+\gamma_{m} f_{m}(u(t)) \\
& =\sum_{j=1}^{m} \gamma_{j} f_{j}(u(t))=f(u(t)) \gamma
\end{aligned}
$$

where the nonlinear block $f(u(t)):=\left[f_{1}(u(t)), f_{2}(u(t)), \cdots\right.$, $\left.f_{m}(u(t))\right] \in \mathbb{R}^{1 \times m}$ and $\gamma:=\left[\gamma_{1}, \gamma_{2}, \cdots, \gamma_{m}\right]^{\mathrm{T}} \in \mathbb{R}^{m}$. The superscript $\mathrm{T}$ denotes the matrix/vector transpose.

It is worth noting that the model in (1) can represent various input nonlinear systems, e.g., when $G(z)=B(z) / A(z)$, and the system is corrupted by the colored noise, i.e., $N(z)=$ $D(z)$, system (1) denotes an input nonlinear equation-error moving average (IN-EEMA) system,

$$
A(z) y(t)=B(z) \bar{u}(t)+D(z) v(t)
$$

where

$$
\begin{aligned}
& A(z):=1+a_{1} z^{-1}+a_{2} z^{-2}+\cdots+a_{n_{a}} z^{-n_{a}}, \\
& B(z):=b_{0}+b_{1} z^{-1}+b_{2} z^{-2}+\cdots+b_{n_{b}} z^{-n_{b}}, \\
& D(z):=1+d_{1} z^{-1}+d_{2} z^{-2}+\cdots+d_{n_{d}} z^{-n_{d}} .
\end{aligned}
$$

Recently, a key variable separation-based (multiinnovation) Newton iterative algorithm has been proposed for nonlinear finite response moving average systems [22] and an identification model-based (multi-innovation) Newton iterative algorithm has been proposed for nonlinear finite response autoregressive moving average systems [23]. On the basis of the works in $[22,23]$, this paper studies several Newton iterative parameter estimation methods for IN-EEMA systems.

The objective of this work is to develop algorithms for improving the computational efficiency and achieving accurate parameter estimation for system (3). We develop three extended Newton iterative parameter estimation algorithms using the key term separation principle and the decomposition technique. The simulation results show that the algorithms are effective for identifying the proposed systems.

Briefly, this paper is organised as follows. Section 2 derives the identification model and develops an extended Newton iterative identification algorithm. Section 3 proposes a key term separation-based extended Newton iterative algorithm. Section 4 presents a key term separation-based extended Newton iterative algorithm using the decomposition technique. Section 5 provides an illustrative example to show the effectiveness of the algorithms. Finally, concluding remarks are offered in Section 6.

\section{The Extended Newton Iterative Identification Algorithm}

For the identification of Hammerstein system, in order to reduce the sensitivity of the projection algorithm to noise and to improve convergence rates of the stochastic gradient algorithm, Ding et al. proposed a Newton recursive and a Newton iterative identification algorithms by using the Newton method (Newton-Raphson method) [35]. Based on the work in [35], this paper considers identification of input nonlinear systems with colored noise and the disturbance is an autoregressive moving average process. Consider system (3) and define the parameter vectors $a$, $b, d, \gamma$, and $\eta$ as

$$
\begin{aligned}
a & :=\left[a_{1}, a_{2}, \cdots, a_{n_{a}}\right]^{\mathrm{T}} \in \mathbb{R}^{n_{a}}, \\
b & :=\left[b_{0}, b_{1}, b_{2}, \cdots, b_{n_{b}}\right]^{\mathrm{T}} \in \mathbb{R}^{n_{b}+1}, \\
d & :=\left[d_{1}, d_{2}, \cdots, d_{n_{d}}\right]^{\mathrm{T}} \in \mathbb{R}^{n_{d}}, \\
\gamma & :=\left[\gamma_{1}, \gamma_{2}, \cdots, \gamma_{m}\right]^{\mathrm{T}} \in \mathbb{R}^{m}, \\
\eta & :=\left[a_{1}, a_{2}, \cdots, a_{n_{a}}, d_{1}, d_{2}, \cdots, d_{n_{d}}\right]^{\mathrm{T}} \in \mathbb{R}^{n_{a}+n_{d}},
\end{aligned}
$$


and the information vector $\varphi(t)$ and the information matrix $F(t)$ as

$$
\begin{aligned}
\varphi(t):= & {\left[-y(t-1),-y(t-2), \cdots,-y\left(t-n_{a}\right),\right.} \\
& \left.v(t-1), \cdots, v\left(t-n_{d}\right)\right]^{\mathrm{T}} \in \mathbb{R}^{n_{a}+n_{d}}, \\
F(t):= & {\left[f(u(t)), f(u(t-1)), \cdots, f\left(u\left(t-n_{b}\right)\right)\right]^{\mathrm{T}} \in \mathbb{R}^{\left(n_{b}+1\right) \times m} . }
\end{aligned}
$$

The output of system (3) can be expressed as

$$
\begin{aligned}
y(t) & =(1-A(z)) y(t)+B(z) \bar{u}(t)+D(z) v(t) \\
& =-\sum_{i=1}^{n_{a}} a_{i} y(t-i)+\sum_{i=0}^{n_{b}} b_{i} \bar{u}(t-i)+\sum_{i=1}^{n_{d}} d_{i} v(t-i)+v(t) \\
& =\varphi^{\mathrm{T}}(t) \eta+b^{\mathrm{T}} F(t) \gamma+v(t) .
\end{aligned}
$$

Assume that the input/output data is $\{u(t), y(t): t=1,2$, $\cdots, L\}$, where $L$ denotes the data length. Define the stacked matrices:

$$
\begin{aligned}
Y(L) & :=[y(1), y(2), \cdots, y(L)]^{\mathrm{T}} \in \mathbb{R}^{L}, \\
\boldsymbol{\Phi}_{0}(L):= & {[\varphi(1), \varphi(2), \cdots, \varphi(L)]^{\mathrm{T}} \in \mathbb{R}^{L \times\left(n_{a}+n_{d}\right),} } \\
\boldsymbol{\Phi}(\gamma) & :=\left[\begin{array}{c}
\gamma^{\mathrm{T}} F^{\mathrm{T}}(1) \\
\gamma^{\mathrm{T}} F^{\mathrm{T}}(2) \\
\vdots \\
\gamma^{\mathrm{T}} F^{\mathrm{T}}(L)
\end{array}\right] \in \mathbb{R}^{L \times\left(n_{b}+1\right),} \\
\boldsymbol{\Psi}(b) & :=\left[\begin{array}{c}
b^{\mathrm{T}} F^{\mathrm{T}}(1) \\
b^{\mathrm{T}} F^{\mathrm{T}}(2) \\
\vdots \\
b^{\mathrm{T}} F^{\mathrm{T}}(L)
\end{array}\right] \in \mathbb{R}^{L \times m} .
\end{aligned}
$$

Define the cost function:

$$
\begin{aligned}
J(\theta) & =J(\eta, b, \gamma):=\left\|Y(L)-\boldsymbol{\Phi}_{0}(L) \eta-\boldsymbol{\Phi}(\gamma) b\right\|^{2} \\
& =\left\|Y(L)-\boldsymbol{\Phi}_{0}(L) \eta-\boldsymbol{\Psi}(b) \gamma\right\|^{2} .
\end{aligned}
$$

The Hessian matrix of $J(\theta)$ is computed by

$$
\begin{aligned}
& H[J(\eta, b, \gamma)] \\
& :=\left[\begin{array}{lll}
\boldsymbol{\Psi}_{0}^{\mathrm{T}}(L) \boldsymbol{\Phi}_{0}(L) & \boldsymbol{\Phi}_{0}^{\mathrm{T}}(L) \boldsymbol{\Phi}(\gamma) & \boldsymbol{\Phi}_{0}^{\mathrm{T}}(L) \boldsymbol{\Psi}(b) \\
\boldsymbol{\Phi}^{\mathrm{T}}(\gamma) \boldsymbol{\Phi}_{0}(L) & \boldsymbol{\Phi}^{\mathrm{T}}(\gamma) \boldsymbol{\Phi}(\gamma) & M(\eta, b, \gamma) \\
\boldsymbol{\Psi}^{\mathrm{T}}(b) \boldsymbol{\Phi}_{0}(L) & M^{\mathrm{T}}(\eta, b, \gamma) & \boldsymbol{\Psi}^{\mathrm{T}}(b) \boldsymbol{\Psi}(b)
\end{array}\right] \in \mathbb{R}^{n \times n},
\end{aligned}
$$

Let $k$ be an iterative variable,

$$
\widehat{\theta}_{k}:=\left[\begin{array}{l}
\widehat{\eta}_{k} \\
\widehat{b}_{k} \\
\widehat{\gamma}_{k}
\end{array}\right]
$$

be the iterative estimate of

$$
\theta:=\left[\begin{array}{l}
\eta \\
b \\
\gamma
\end{array}\right]
$$

at the $k$ th iteration. Using the similar method in [35], minimizing $J(\theta)$, we have

$$
\begin{aligned}
\widehat{\theta}_{k}= & \widehat{\theta}_{k-1}\left\{H\left[J\left(\widehat{\eta}_{k-1}, \widehat{b}_{k-1}, \widehat{\gamma}_{k-1}\right)\right]\right\}^{-1} \\
& \times \operatorname{grad}_{\theta}\left[J\left(\widehat{\eta}_{k-1}, \widehat{b}_{k-1}, \widehat{\gamma}_{k-1}\right)\right] .
\end{aligned}
$$

Here, the question is that it is impossible to accomplish computing $\widehat{\theta}_{k}$ because the unmeasurable noise $v_{k}(t-i), i=1$, $2, \ldots, n_{d}$, contained in $\varphi(t)$ leads to an unknown $\Phi_{0}$ in $H\left[J\left(\widehat{\eta}_{k-1}, \widehat{b}_{k-1}, \widehat{\gamma}_{k-1}\right)\right]$. Inspired by the former work in [36, 37], these unknown issues can be dealt with by replacing the unknown items with their estimates. Let the iterative estimate of $\varphi(t)$ be $\widehat{\varphi}_{k}(t)$ :

$$
\begin{gathered}
\widehat{\varphi}_{k}(t):=\left[-y(t-1),-y(t-2), \cdots,-y\left(t-n_{a}\right), \widehat{v}(t-1),\right. \\
\left.\widehat{v}(t-2), \cdots, \widehat{v}_{k-1}\left(t-n_{d}\right)\right]^{\mathrm{T}} .
\end{gathered}
$$

According to (7), we have

$$
v(t)=y(t)-\varphi^{\mathrm{T}} \eta-b^{\mathrm{T}} F(t) \gamma
$$

Replacing the unknown parameters and vectors in the above equation with their iterative estimates $\widehat{\eta}_{k}, \widehat{b}_{k}, \widehat{\gamma}$, and $\widehat{\varphi}_{k}(t)$, we can compute $\widehat{v}_{k}(t-i)$ through

$$
\widehat{v}_{k}(t)=y(t-i)-\widehat{\varphi}_{k}^{\mathrm{T}}(t-i) \widehat{\eta}_{k}-\widehat{b}_{k}^{\mathrm{T}} F(t-i) \widehat{\gamma}_{k} .
$$

Then the extended Newton iterative (E-NI) algorithm for IN-EEMA systems is summarized as follows:

$$
\widehat{\theta}_{k}=\widehat{\theta}_{k-1}+\widehat{\boldsymbol{\Omega}}_{k}^{-1}\left[\begin{array}{c}
\widehat{\boldsymbol{\Phi}}_{0}^{\mathrm{T}}(L) \\
\boldsymbol{\Phi}^{\mathrm{T}}\left(\widehat{\gamma}_{k-1}\right) \\
\boldsymbol{\Psi}^{\mathrm{T}}\left(\widehat{b}_{k-1}\right)
\end{array}\right]\left[Y(L)-\widehat{\boldsymbol{\Phi}}_{0}(L) \widehat{\eta}_{k-1}-\boldsymbol{\Phi}\left(\widehat{\gamma}_{k-1}\right) \widehat{b}_{k-1}\right],
$$

where $n:=n_{a}+n_{d}+\left(n_{b}+1\right)+m$. 


$$
\begin{aligned}
& \widehat{\boldsymbol{\Omega}}_{k}=\left[\begin{array}{ccc}
\widehat{\boldsymbol{\Phi}}_{0}^{\mathrm{T}}(L) \widehat{\boldsymbol{\Phi}}_{0}(L) & \widehat{\boldsymbol{\Phi}}_{0}^{\mathrm{T}}(L) \boldsymbol{\Phi}\left(\widehat{\gamma}_{k-1}\right) & \widehat{\boldsymbol{\Phi}}_{0}^{\mathrm{T}}(L) \boldsymbol{\Psi}\left(\widehat{b}_{k-1}\right) \\
\widehat{\boldsymbol{\Phi}}^{\mathrm{T}}\left(\widehat{\gamma}_{k-1}\right) \widehat{\boldsymbol{\Phi}}_{0}(L) & \boldsymbol{\Phi}^{\mathrm{T}}\left(\widehat{\gamma}_{k-1}\right) \boldsymbol{\Phi}\left(\widehat{\gamma}_{k-1}\right) & \widehat{M}\left(\widehat{\theta}_{k-1}\right) \\
\boldsymbol{\Psi}^{\mathrm{T}}\left(\widehat{b}_{k-1}\right) \widehat{\boldsymbol{\Phi}}_{0}(L) & \widehat{M}^{\mathrm{T}}\left(\widehat{\theta}_{k-1}\right) & \boldsymbol{\Psi}^{\mathrm{T}}\left(\widehat{b}_{k-1}\right) \boldsymbol{\Psi}\left(\widehat{b}_{k-1}\right)
\end{array}\right] \\
& \widehat{M}\left(\widehat{\theta}_{k-1}\right)=\sum_{t-1}^{L}\left\{F(t)\left[-y(t)+\widehat{\varphi}^{\mathrm{T}}(t) \widehat{\eta}_{k-1}+\widehat{b}_{k-1}^{\mathrm{T}} F(t) \widehat{\gamma}_{k-1}\right]\right\} \\
& +\boldsymbol{\Phi}^{\mathrm{T}}\left(\widehat{\gamma}_{k-1}\right) \Psi\left(\widehat{b}_{k-1}\right), \\
& Y(L)=[y(1), y(2), \cdots, y(L)]^{\mathrm{T}}, \\
& \widehat{\boldsymbol{\Phi}}_{0}(L)=\left[\widehat{\varphi}_{k}(1), \widehat{\varphi}_{k}(2), \cdots, \widehat{\varphi}_{k}(L)\right]^{\mathrm{T}}, \\
& \Psi\left(\widehat{b}_{k-1}\right)=\left[F^{\mathrm{T}}(1) \widehat{b}_{k-1}, F^{\mathrm{T}}(2) \widehat{b}_{k-1}, \cdots, F^{\mathrm{T}}(L) \widehat{b}_{k-1}\right]^{\mathrm{T}}, \\
& \boldsymbol{\Phi}\left(\widehat{\gamma}_{k-1}\right)=\left[F(1) \widehat{\gamma}_{k-1}, F(2) \widehat{\gamma}_{k-1}, \cdots, F(L) \widehat{\gamma}_{k-1}\right]^{\mathrm{T}}, \\
& \widehat{\varphi}_{k}(t)=\left[-y(t-1),-y(t-2), \cdots,-y\left(t-n_{a}\right),\right. \\
& \left.\widehat{v}(t-1), \widehat{v}(t-2), \cdots, \widehat{v}_{k-1}\left(t-n_{d}\right)\right]^{\mathrm{T}}, \\
& F(t)=\left[\begin{array}{cccc}
f_{1}(u(t)) & f_{2}(u(t)) & \cdots & f_{m}(u(t)) \\
f_{1}(u(t-1)) & f_{2}(u(t-1)) & \cdots & f_{m}(u(t-1)) \\
\vdots & \vdots & & \vdots \\
f_{1}\left(u\left(t-n_{b}\right)\right) & f_{2}\left(u\left(t-n_{b}\right)\right) & \cdots & f_{m}\left(u\left(t-n_{b}\right)\right)
\end{array}\right], \\
& \widehat{v}_{k}(t)=y(t-i)-\widehat{\varphi}_{k}^{\mathrm{T}}(t-i) \widehat{\eta}_{k}-\widehat{b}_{k}^{\mathrm{T}} F(t-i) \widehat{\gamma}_{k} .
\end{aligned}
$$

Notice that the Hessian matrix in (18) requires more computational effort as the number of system parameters grows.

\section{The Key Term Separation-Based Extended Newton Iterative Algorithm}

In this section, the key term separation principle is employed to parameterize the IN-EEMA systems. The core idea of the key term separation technique is to express the system output as a linear combination of the system parameters $[38,39]$. Therefore, the redundant parameter estimation can be avoided. Let the first parameter of $B(z)$ be 1 , and the system in (7) can be rewrite as

$y(t)=-\sum_{i=1}^{n_{a}} a_{i} y(t-i)+\sum_{i=1}^{n_{b}} b_{i} \bar{u}(t-i)+\sum_{i=1}^{n_{d}} d_{i} v(t-i)+v(t)$.

Substituting the key term $u(t)$ into (2) gives

$$
\begin{aligned}
y(t)= & -\sum_{i=1}^{n_{a}} a_{i} y(t-i)+\sum_{j=1}^{m} \gamma_{i} f_{i}(u(t))+\sum_{i=1}^{n_{b}} b_{i} \bar{u}(t-i) \\
& +\sum_{i=1}^{n_{d}} d_{i} v(t-i)+v(t) .
\end{aligned}
$$

Define the parameter vector $\vartheta$ and the information vectors $\varphi_{a}(t), \varphi_{d}(t)$, and $\phi(t)$ as

$$
\begin{aligned}
\vartheta:= & {\left[a^{\mathrm{T}}, \gamma^{\mathrm{T}}, b_{1}, b_{2}, \cdots, b_{n_{b}}, d^{\mathrm{T}}\right]^{\mathrm{T}} \in \mathbb{R}^{n}, \quad n=n_{a}+n_{b}+m, } \\
\varphi_{a}(t):= & {\left[-y(t-1),-y(t-2), \cdots, y\left(n-n_{a}\right)\right] \in \mathbb{R}^{n_{a}}, } \\
\varphi_{d}(t):= & {\left[v(t-1), v(t-2), \cdots, v\left(t-n_{d}\right)\right] \in \mathbb{R}^{n_{d}}, } \\
\phi(t):= & {\left[\varphi_{a}^{\mathrm{T}}(t), f(u(t)), \bar{u}(t-1), \bar{u}(t-2), \cdots, \bar{u}\left(t-n_{b}\right),\right.} \\
& \left.\varphi_{d}^{\mathrm{T}}(t)\right]^{\mathrm{T}} \in \mathbb{R}^{n} .
\end{aligned}
$$

Then system (3) takes the form of the identification model as

$$
y(t)=\phi^{\mathrm{T}}(t) \vartheta+v(t) .
$$

Consider $L$ sets of data and define the stacked vector $Y(L)$ and the stacked matrix $\boldsymbol{\Phi}(L)$ as

$$
\begin{gathered}
Y(L):=\left[\begin{array}{c}
y(1) \\
y(2) \\
\vdots \\
y(L)
\end{array}\right] \in \mathbb{R}^{L}, \\
\Phi(L):=\left[\begin{array}{c}
\phi(1) \\
\phi(2) \\
\vdots \\
\phi(L)
\end{array}\right] \in \mathbb{R}^{L \times n} .
\end{gathered}
$$

Define the cost function

$$
J(\vartheta):=\|Y(L)-\Phi(L) \vartheta\|^{2} .
$$

Let $\widehat{\vartheta}_{k}$ be the iterative estimation of $\vartheta$ at the $k$ th iteration, and using the Newton method to minimize $J(9)$ gives

$$
\begin{aligned}
\widehat{\vartheta}_{k} & =\widehat{\vartheta}_{k-1}-\left\{H\left(J\left(\widehat{\vartheta}_{k-1}\right)\right)\right\}^{-1} \operatorname{grad}\left[J\left(\widehat{\vartheta}_{k-1}\right)\right] \\
& =\widehat{\vartheta}_{k-1}+\left[\boldsymbol{\Phi}^{\mathrm{T}}(L) \boldsymbol{\Phi}(L)\right]^{-1} \boldsymbol{\Phi}^{\mathrm{T}}(L)\left[Y(L)-\boldsymbol{\Phi}(L) \widehat{\vartheta}_{k-1}\right] .
\end{aligned}
$$

Replace the unknown variables $\bar{u}(t-i)$ at the $k$ th iteration with the iterative estimates $\widehat{u}_{k-1}(t-i)$ and define

$$
\begin{aligned}
\widehat{\phi}_{k}(t):= & {\left[\varphi_{a}^{\mathrm{T}}(t), f(u(t)), \widehat{\bar{u}}_{k-1}(t-1), \widehat{\bar{u}}_{k-1}(t-2), \cdots,\right.} \\
& \left.\widehat{\bar{u}}_{k-1}\left(t-n_{b}\right), \widehat{\varphi}_{d, k-1}^{\mathrm{T}}(t)\right]^{T} \in \mathbb{R}^{n} \\
\widehat{\vartheta}_{k}:= & {\left[a_{k}^{\mathrm{T}}, \gamma_{k}^{\mathrm{T}}, \widehat{b}_{1, k}, \widehat{b}_{2, k}, \cdots, \widehat{b}_{n_{b}, k}, \widehat{d}_{k}^{\mathrm{T}}\right]^{\mathrm{T}} \in \mathbb{R}^{n} }
\end{aligned}
$$


Replacing $\widehat{\gamma}_{i, k}$ in (2) with $\gamma_{i}$, the iterative estimates of $\bar{u}(t-i)$ can be computed through

$$
\begin{aligned}
\widehat{\hat{u}}(t-i) & =\widehat{\gamma}_{1, k} f_{1}(u(t-i))+\widehat{\gamma}_{2, k} f_{2}(u(t-i))+\cdots+\widehat{\gamma}_{m, k} f_{m}(u(t-i)) \\
& =\sum_{j=1}^{m} \widehat{\gamma}_{j, k} f_{j}(u(t-i)) f(u(t-i)) \widehat{\gamma}_{k} .
\end{aligned}
$$

From (28), we have

$$
v(t-i)=y(t-i)-\phi^{\mathrm{T}}(t-i) \vartheta
$$

Replacing $\phi(t-i)$ and $\vartheta$ with $\widehat{\phi}_{k}(t-i)$ and $\widehat{\vartheta}_{k}$ respectively, the iterative estimates of $v(t-i)$ is computed by

$$
\widehat{v}_{k}(t-i)=y(t-i)-\widehat{\phi}_{k}^{\mathrm{T}}(t-i) \widehat{\vartheta}_{k}
$$

Substituting unknown $\phi(t)$ in the stacked matrix $\boldsymbol{\Phi}(L)$ with its estimate, the iterative estimate of $\Phi(L)$ is given by

$$
\widehat{\boldsymbol{\Phi}}(L):=\left[\widehat{\phi}_{k}(1), \widehat{\phi}_{k}(2), \cdots, \widehat{\phi}_{k}(L)\right]^{\mathrm{T}} \in \mathbb{R}^{L \times n} .
$$

Then we replace the $\Phi(L)$ in (33) with $\widehat{\Phi}(L)$ and summarize the key term separation-based extended Newton iterative (KT-NI) algorithm for the IN-EEMA model:

$$
\begin{aligned}
\widehat{\vartheta}_{k}= & \widehat{\vartheta}_{k-1}+\left[\widehat{\boldsymbol{\Phi}}_{k}^{\mathrm{T}}(L) \widehat{\boldsymbol{\Phi}}_{k}(L)\right]^{-1} \widehat{\boldsymbol{\Phi}}_{k}^{\mathrm{T}}(L)\left[Y(L)-\widehat{\boldsymbol{\Phi}}_{k}(L) \widehat{\vartheta}_{k-1}\right] \\
Y(L)= & {[y(1), y(2), \cdots, y(L)]^{\mathrm{T}}, } \\
\widehat{\boldsymbol{\Phi}}_{k}(L)= & {\left[\widehat{\phi}_{k}(1), \widehat{\phi}_{k}(2), \cdots, \widehat{\phi}_{k}(L)\right]^{\mathrm{T}}, } \\
\widehat{\phi}_{k}(t)= & {\left[\varphi_{a}^{\mathrm{T}}(t), f(u(t)), \widehat{\bar{u}}_{k-1}(t-1), \widehat{\hat{u}}_{k-1}(t-2), \cdots,\right.} \\
& \left.\widehat{\bar{u}}_{k-1}\left(t-n_{b}\right), \widehat{\varphi}_{d, k-1}^{\mathrm{T}}(t)\right]^{\mathrm{T}}, \\
\varphi_{a}(t)= & {\left[-y(t-1),-y(t-2), \cdots,-y\left(t-n_{a}\right)\right]^{\mathrm{T}}, } \\
f(u(t))= & {\left[f_{1}(u(t)), f_{2}(u(t)), \cdots, f_{m}(u(t))\right], } \\
\widehat{\varphi}_{d, k}(t)= & {\left[\widehat{v}_{k}(t-1), \widehat{v}_{k}(t-2), \cdots, \widehat{v}_{k}\left(t-n_{d}\right)\right]^{\mathrm{T}}, } \\
\widehat{\bar{u}}_{k}(t-i)= & f(u(t-i)) \widehat{\gamma}_{k}, \\
\widehat{v}_{k}(t-i)= & y(t-i)-\widehat{\phi}_{k}^{\mathrm{T}}(t-i) \widehat{\vartheta}_{k}, \\
\widehat{\vartheta}_{k}(t)= & {\left[\widehat{a}_{1, k}, \widehat{a}_{2, k}, \cdots, \widehat{a}_{n, k}, \widehat{\gamma}_{k}^{\mathrm{T}}, \widehat{b}_{1, k}, \widehat{b}_{2, k}, \cdots, \widehat{b}_{n_{b}, k} \widehat{d}_{1, k}, \widehat{d}_{2, k}, \cdots, \widehat{d}_{n_{d}}, k\right]^{\mathrm{T}}, }
\end{aligned}
$$

$$
\widehat{\gamma}_{k}=\left[\widehat{\gamma}_{1, k}, \widehat{\gamma}_{2, k}, \cdots, \widehat{\gamma}_{m, k}\right]^{\mathrm{T}} .
$$

\section{Extended Newton Iterative Algorithm Using the Decomposition Technique}

Define the information vectors $\varphi_{a}(t)$ and $\varphi_{d}(t)$ as

$$
\begin{aligned}
\varphi_{a}(t) & :=\left[-y(t-1),-y(t-2), \cdots,-y\left(t-n_{a}\right)\right]^{\mathrm{T}} \in \mathbb{R}^{n_{a}}, \\
\varphi_{d}(t) & :=\left[v(t-1), v(t-2), \cdots, v\left(t-n_{d}\right)\right]^{\mathrm{T}} \in \mathbb{R}^{n_{d}} .
\end{aligned}
$$

Rewrite the IN-EEMA system in (7) as

$$
\begin{aligned}
y(t) & =(1-A(z)) y(t)+B(z) \bar{u}(t)+D(z) v(t) \\
& =-\sum_{i=1}^{n_{a}} a_{i} y(t-i)+\sum_{i=0}^{n_{b}} b_{i} \bar{u}(t-i)+\sum_{i=1}^{n_{d}} d_{i} v(t-i)+v(t) \\
& =\varphi_{a}^{\mathrm{T}}(t) a+b^{\mathrm{T}} F(t) \gamma+\varphi_{d}^{\mathrm{T}}(t) d+v(t) .
\end{aligned}
$$

By applying the decomposition technique to the INEEMA system, we divide model (51) into two subsystems. One subsystem contains the system parameter vector $\vartheta_{1}:=[a, b]^{\mathrm{T}}$, and the other subsystem contains the system parameter vector $\vartheta_{2}:=[\gamma, d]^{\mathrm{T}}$. Define two auxiliary outputs:

$$
\begin{aligned}
& y_{1}(t):=y(t)-\varphi_{d}^{\mathrm{T}}(t) d, \\
& y_{2}(t):=y(t)-\varphi_{a}^{\mathrm{T}}(t) a .
\end{aligned}
$$

Combining (51), (52), and (53), it gives

$$
\begin{aligned}
& S_{1}: y_{1}(t)=\left[\varphi_{a}^{\mathrm{T}}(t), \gamma^{\mathrm{T}} F^{\mathrm{T}}(t)\right] \vartheta_{1}+v(t), \\
& S_{2}: y_{2}(t)=\left[b^{\mathrm{T}} F^{\mathrm{T}}(t), \varphi_{d}^{\mathrm{T}}(t)\right] \vartheta_{2}+v(t) .
\end{aligned}
$$

Let $\widehat{\vartheta}_{1, k}:=\left[\widehat{a}_{k}, \widehat{b}_{k}\right]^{\mathrm{T}}$ and $\widehat{\vartheta}_{2, k}:=\left[\widehat{\gamma}_{k}, \widehat{d}_{k}\right]^{\mathrm{T}}$ be the iterative estimates of $\vartheta_{1}$ and $\vartheta_{2}$ at the $k$ th iteration, respectively. Define the cost functions:

$$
\begin{aligned}
J_{1}\left(\vartheta_{1}\right):= & \left.J\left(\vartheta_{1}, \widehat{\vartheta}_{2, k-1}\right)=\sum_{t=1}^{L}\left\{y_{1}(t)-\left[\varphi_{a}^{\mathrm{T}}(t), \widehat{\gamma}_{k-1}^{\mathrm{T}} F(t)\right] \vartheta_{1}\right]\right\}^{2} \\
& \cdot \sum_{t=1}^{L}\left[y(t)-\varphi_{a}^{\mathrm{T}}(t) a-b^{\mathrm{T}} F^{\mathrm{T}}(t) \widehat{\gamma}_{k-1}-\varphi_{d}^{\mathrm{T}}(t) \widehat{d}_{k-1}\right]^{2}, \\
J_{2}\left(\vartheta_{2}\right):= & \left.J\left(\widehat{\vartheta}_{1, k}, \vartheta_{2}\right)=\sum_{t=1}^{L}\left\{y_{2}(t)-\left[b_{k}^{\mathrm{T}} F(t), \varphi_{d}^{\mathrm{T}}(t)\right] \vartheta_{2}\right]\right\}^{2} \\
& \cdot \sum_{t=1}^{L}\left[y(t)-\varphi_{a}^{\mathrm{T}}(t) a-b_{k}^{\mathrm{T}} F^{\mathrm{T}}(t) \gamma-\varphi_{d}^{\mathrm{T}}(t) d\right]^{2} .
\end{aligned}
$$


Notice that the unknown parameter vectors $\vartheta_{1}$ and $\vartheta_{2}$ in cost functions above are replaced by their iterative estimates. For the sake of brevity, define the stacked matrices:

$$
\begin{aligned}
\Gamma(L):=\left[\begin{array}{c}
\varphi_{a}^{\mathrm{T}}(1) \\
\varphi_{a}^{\mathrm{T}}(2) \\
\vdots \\
\varphi_{a}^{\mathrm{T}}(L)
\end{array}\right] \in \mathbb{R}^{L \times n_{a}}, \\
\boldsymbol{\Phi}(\gamma, L):=\left[\begin{array}{cc}
\varphi_{a}^{\mathrm{T}}(1) & \gamma^{\mathrm{T}} F^{\mathrm{T}}(1) \\
\varphi_{a}^{\mathrm{T}}(2) & \gamma^{\mathrm{T}} F^{\mathrm{T}}(2) \\
\vdots & \vdots \\
\varphi_{a}^{\mathrm{T}}(L) & \gamma^{\mathrm{T}} F^{\mathrm{T}}(L)
\end{array}\right] \in \mathbb{R}^{L \times\left(n_{a}+n_{b}+1\right)}, \\
\boldsymbol{\Omega}(L):=\left[\begin{array}{cc}
\varphi_{d}^{\mathrm{T}}(1) \\
\varphi_{d}^{\mathrm{T}}(2) \\
\vdots \\
\varphi_{d}^{\mathrm{T}}(L)
\end{array}\right] \in \mathbb{R}^{L \times n_{d}}, \\
\boldsymbol{\Psi}(b, L):=\left[\begin{array}{cc}
b^{\mathrm{T}} F(1) & \varphi_{d}^{\mathrm{T}}(1) \\
b^{\mathrm{T}} F(2) & \varphi_{d}^{\mathrm{T}}(2) \\
\vdots & \vdots \\
b^{\mathrm{T}} F(L) & \varphi_{d}^{\mathrm{T}}(L)
\end{array}\right] \in \mathbb{R}^{L \times\left(n_{a}+n_{b}+1\right)} .
\end{aligned}
$$

Then the cost functions can be rewritten as

$$
\begin{aligned}
& J_{1}\left(\vartheta_{1}\right)=\left\|Y(L)-\boldsymbol{\Phi}(\gamma, L) \vartheta_{1}-\boldsymbol{\Omega}(L) d\right\|^{2}, \\
& J_{2}\left(\vartheta_{2}\right)=\left\|Y(L)-\Gamma(L) a-\Psi(b, L) \vartheta_{2}\right\|^{2} .
\end{aligned}
$$

Minimizing $J_{1} \vartheta_{1}$ and $J_{2}\left(\vartheta_{2}\right)$ by using the Newton method, it yields

$$
\begin{aligned}
\widehat{\vartheta}_{1, k}= & \widehat{\vartheta}_{1, k-1}-\left\{H\left[J_{1}\left(\widehat{\vartheta}_{1, k-1}\right)\right]\right\}^{-1} \operatorname{grad}\left[J_{1}\left(\widehat{\vartheta}_{1, k-1}\right)\right] \\
= & \widehat{\vartheta}_{1, k-1}+\left[\boldsymbol{\Phi}^{\mathrm{T}}(\gamma, L) \boldsymbol{\Psi}(\gamma, L)\right]^{-1} \boldsymbol{\Psi}^{\mathrm{T}}(\gamma, L) \\
& \times\left[Y(L)-\boldsymbol{\Phi}(\gamma, L) \widehat{\vartheta}_{1, k-1}-\boldsymbol{\Omega}(L) d\right], \\
\widehat{\vartheta}_{2, k}= & \widehat{\vartheta}_{2, k-1}-\left\{H\left[J_{2}\left(\widehat{\vartheta}_{2, k-1}\right)\right]\right\}^{-1} \operatorname{grad}\left[J_{2}\left(\widehat{\vartheta}_{2, k-1}\right)\right] \\
= & \widehat{\vartheta}_{2, k-1}+\left[\boldsymbol{\Psi}^{\mathrm{T}}(b, L) \boldsymbol{\Psi}(b, L)\right]^{-1} \boldsymbol{\Psi}^{\mathrm{T}}(b, L) \\
& \times\left[Y(L)-\boldsymbol{\Gamma}(L) a-\boldsymbol{\Psi}(b, L) \widehat{\vartheta}_{2, k-1}\right] .
\end{aligned}
$$

The similar issue arises that the information vector $\varphi_{d}(t)$ contains the unknown items $v(t-i)$; hence, $\widehat{\vartheta}_{1, k}$ and $\widehat{\vartheta}_{2, k}$ in the two equations above cannot be directly calculated. Here, following the solution carried out in the last two sections and replacing the unknown noise items with their iterative estimates $\widehat{v}_{k-1}(t-i)$ at iteration $k-1$, the iterative estimate of $\varphi_{d}(t)$ can be represented as

$$
\widehat{\varphi}_{d, k}(t):=\left[\widehat{v}_{k-1}(t-1), \widehat{v}_{k-1}(t-2), \cdots, \widehat{v}_{k-1}\left(t-n_{d}\right)\right]^{\mathrm{T}} \in \mathbb{R}^{n_{d}} .
$$

From (51), we have

$$
v(t)=y(t)-\varphi_{a}^{\mathrm{T}} a-b^{\mathrm{T}} F(t) \gamma-\varphi_{d}^{\mathrm{T}}(t) d
$$

Replacing unknown vectors $a, b, \gamma$, and $\varphi_{d}(t)$ with their iterative estimates $\widehat{a}_{k}, \widehat{b}_{k}, \widehat{\gamma}_{k}$, and $\widehat{\varphi}_{d, k}(t)$, the estimated noise $\widehat{v}_{k}(t)$ can be calculated from

$$
\widehat{v}_{k}(t)=y(t)-\varphi_{a}^{\mathrm{T}}(t) \widehat{a}_{k}-b_{k}^{\mathrm{T}} F(t) \widehat{\gamma}_{k}-\widehat{\varphi}_{d, k}^{\mathrm{T}}(t) \widehat{d}_{k} .
$$

Substituting the estimated vectors $\widehat{\gamma}_{k-1}, \widehat{d}_{k-1}, \widehat{a}_{k}$, and $\widehat{b}_{k}$ for the unknown vectors $\gamma, d, a$, and $b$ and substituting $\widehat{\Psi}^{\mathrm{T}}\left(\widehat{b}_{k}, L\right)$ and $\widehat{\boldsymbol{\Omega}}(L)$ for $\boldsymbol{\Psi}^{\mathrm{T}}(b, L)$ and $\boldsymbol{\Omega}(L)$, respectively, we can summarize the decomposition-based extended Newton iterative (D-KT-NI) identification algorithm for IN-EEMA systems i.e., the hierarchical extended Newton iterative algorithm

$$
\begin{aligned}
& \widehat{\vartheta}_{1, k}=\widehat{\vartheta}_{1, k-1}+\left[\widehat{\boldsymbol{\Phi}}^{\mathrm{T}}\left(\widehat{\gamma}_{k-1}, L\right) \widehat{\boldsymbol{\Phi}}^{\mathrm{T}}\left(\widehat{\gamma}_{k-1}, L\right)\right]^{-1} \widehat{\boldsymbol{\Phi}}^{\mathrm{T}}\left(\widehat{\gamma}_{k-1}, L\right) \\
& \cdot\left[Y(L)-\widehat{\boldsymbol{\Phi}}^{\mathrm{T}}\left(\widehat{\gamma}_{k-1}, L\right) \widehat{\vartheta}_{1, k-1}-\widehat{\boldsymbol{\Omega}}_{k}(L) \widehat{d}_{k-1}\right] \text {, } \\
& \widehat{\vartheta}_{2, k}=\widehat{\vartheta}_{2, k-1}+\left[\widehat{\boldsymbol{\Psi}}_{k}^{\mathrm{T}}\left(\widehat{b}_{k}, L\right) \widehat{\boldsymbol{\Psi}}_{k}\left(\widehat{b}_{k}, L\right)\right]^{-1} \widehat{\boldsymbol{\Psi}}_{k}^{\mathrm{T}} \\
& \left(\widehat{b}_{k}, L\right)\left[Y(L)-\boldsymbol{\Gamma}(L) \widehat{a}_{k}-\widehat{\Psi}_{k}\left(\widehat{b}_{k}, L\right) \widehat{\vartheta}_{2, k-1}\right] \text {, } \\
& Y(L)=[y(1), y(2), \cdots, y(L)]^{\mathrm{T}} \text {, } \\
& \Gamma(L)=\left[\varphi_{a}(1), \varphi_{a}(2), \cdots, \varphi_{a}(L)\right]^{\mathrm{T}}, \\
& \Phi\left(\widehat{\gamma}_{k,-1}, L\right)=\left[\begin{array}{cc}
\varphi_{a}^{\mathrm{T}}(1) & \widehat{\gamma}_{k-1}^{\mathrm{T}} F^{\mathrm{T}}(1) \\
\varphi_{a}^{\mathrm{T}}(2) & \widehat{\gamma}_{k-1}^{\mathrm{T}} F^{\mathrm{T}}(2) \\
\vdots & \vdots \\
\varphi_{a}^{\mathrm{T}}(L) & \widehat{\gamma}_{k-1}^{\mathrm{T}} F^{\mathrm{T}}(L)
\end{array}\right], \\
& \Psi\left(\widehat{b}_{k}, L\right)=\left[\begin{array}{cc}
\widehat{b}_{k}^{\mathrm{T}} F(1) & \widehat{\varphi}_{d, k}^{\mathrm{T}}(1) \\
\widehat{b}_{k}^{\mathrm{T}} F(2) & \widehat{\varphi}_{d, k}^{\mathrm{T}}(2) \\
\vdots & \vdots \\
\widehat{b}_{k}^{\mathrm{T}} F(L) & \widehat{\varphi}_{d, k}^{\mathrm{T}}(L)
\end{array}\right], \\
& \widehat{\boldsymbol{\Omega}}(L)=\left[\widehat{\varphi}_{d, k}^{\mathrm{T}}(1), \widehat{\varphi}_{d, k}^{\mathrm{T}}(2), \cdots, \widehat{\varphi}_{d, k}^{\mathrm{T}}(L)\right]^{\mathrm{T}}, \\
& \varphi_{a}(t)=\left[-y(t-1),-y(t-2), \cdots,-y\left(t-n_{a}\right)\right]^{\mathrm{T}}, \\
& \widehat{\varphi}_{d, k}(t)=\left[\widehat{v}_{k}(t-1), \widehat{v}_{k}(t-2), \cdots, \widehat{v}_{k}\left(t-n_{d}\right),\right]^{\mathrm{T}} \text {, }
\end{aligned}
$$


TABLE 1: The parameter estimates and errors of the E-NI algorithm.

\begin{tabular}{cccccccccc}
\hline$\sigma^{2}$ & $k$ & $a_{1}$ & $a_{2}$ & $b_{1}$ & $b_{2}$ & $\gamma_{1}$ & $\gamma_{2}$ & $d_{1}$ \\
\hline & 1 & -1.08845 & 0.71303 & 0.74340 & 0.98758 & 0.58515 & 0.65293 & -0.00146 & 18.66607 \\
& 2 & -1.11208 & 0.71639 & 0.61498 & 0.96651 & 0.49424 & 0.63639 & 0.11693 & 20.41973 \\
& 3 & -1.09042 & 0.70042 & 0.55489 & 0.90946 & 0.45489 & 0.60967 & -0.13144 & 8.02220 \\
$0.10^{2}$ & 4 & -1.09966 & 0.69927 & 0.54109 & 0.89591 & 0.44933 & 0.60349 & -0.28284 & 0.95099 \\
& 5 & -1.10337 & 0.70316 & 0.54118 & 0.89811 & 0.44974 & 0.60455 & -0.30211 & 0.53527 \\
& 10 & -1.10207 & 0.70266 & 0.54220 & 0.89854 & 0.45006 & 0.60491 & -0.30147 & 0.47475 \\
& 15 & -1.10207 & 0.70265 & 0.54220 & 0.89854 & 0.45006 & 0.60491 & -0.30147 & 0.47471 \\
& 20 & -1.10207 & 0.70265 & 0.54220 & 0.89854 & 0.45006 & 0.60491 & -0.30147 & 0.47471 \\
\hline & 1 & -1.07028 & 0.70468 & 0.73066 & 0.99064 & 0.57906 & 0.65907 & -0.01324 & 17.96686 \\
& 2 & -1.06241 & 0.69400 & 0.56781 & 0.90670 & 0.45678 & 0.62211 & -0.19086 & 5.65096 \\
& 3 & -1.09391 & 0.69393 & 0.50848 & 0.87623 & 0.43431 & 0.60337 & -0.29842 & 2.42364 \\
$0.50^{2}$ & 4 & -1.10633 & 0.70643 & 0.51112 & 0.89287 & 0.43789 & 0.60902 & -0.31520 & 2.17016 \\
& 5 & -1.10194 & 0.70632 & 0.51319 & 0.89320 & 0.43879 & 0.60950 & -0.31016 & 1.98764 \\
& 10 & -1.10163 & 0.70488 & 0.51303 & 0.89271 & 0.43855 & 0.60934 & -0.30986 & 1.98664 \\
& 15 & -1.10165 & 0.70490 & 0.51301 & 0.89269 & 0.43856 & 0.60933 & -0.30988 & 1.98745 \\
& 20 & -1.10165 & 0.70490 & 0.51301 & 0.89269 & 0.43856 & 0.60933 & -0.30988 & 1.98744 \\
\hline True values & -1.10000 & 0.70000 & 0.55000 & 0.90000 & 0.45000 & 0.60000 & -0.30000 & \\
\hline
\end{tabular}

$\widehat{v}_{k}(t)=y(t)-\varphi_{a}^{\mathrm{T}}(t) \widehat{a}_{k}-b_{k}^{\mathrm{T}} F(t) \widehat{\gamma}_{k}-\widehat{\varphi}_{d, k}^{\mathrm{T}}(t) \widehat{d}_{k}$,

$$
F(t)=\left[\begin{array}{cccc}
f_{1}(u(t)) & f_{2}(u(t)) & \cdots & f_{m}(u(t)) \\
f_{1}(u(t-1)) & f_{2}(u(t-1)) & \cdots & f_{m}(u(t-1)) \\
\vdots & \vdots & & \vdots \\
f_{1}\left(u\left(t-n_{b}\right)\right) & f_{2}\left(u\left(t-n_{b}\right)\right) & \cdots & f_{m}\left(u\left(t-n_{b}\right)\right)
\end{array}\right] .
$$

The steps for computing the parameter estimates $\widehat{\vartheta}_{1, k}$ and $\widehat{\vartheta}_{2, k}$ in (62), (63), (64), (65), (66), (67), (68), (69), (70), (71), and (72) are as follows.

(1) Set the data length $L$, collect input output data $\{u(t), y(t): t=1,2, \cdots, L\}$, form $Y(L)$, and preset a small threshold value $\epsilon>0$

(2) Let $k=1$, and set the initial values $\widehat{\vartheta}_{1,0}=1_{n_{a}+n_{b}}$, $\widehat{\vartheta}_{2,0}=1_{m+n_{d}}, \widehat{v}(t-i)=a$ random vector, $i=1,2, \ldots$, $n_{d}, p_{0}=10^{6}$

(3) Form $\varphi_{a}(t)$ using (69), $\Gamma(L)$ using (65), and $\Phi\left(\widehat{\gamma}_{k-1}, L\right)$ using (66)

(4) Update $\vartheta_{1, k}$ using (62)

(5) Form $\widehat{\boldsymbol{\Omega}}(L)$ using (68) and $\boldsymbol{\Psi}\left(\widehat{b}_{k}, L\right)$ using (67)

(6) Update $\widehat{\vartheta}_{2, k}$ using (63)

(7) Compute $v_{k}(t-i)$ using (71)
(8) If $\left\|\widehat{\vartheta}_{1, k}-\widehat{\vartheta}_{1, k-1}\right\|+\left\|\widehat{\vartheta}_{2, k}-\widehat{\vartheta}_{2, k-1}\right\|>\epsilon$, increase $k$ by 1 , go to step 4; otherwise, terminate the procedure and obtain $k, \widehat{\vartheta}_{1, k}$, and $\widehat{\vartheta}_{2, k}$

\section{Example}

Consider the following nonlinear simulation system:

$$
\begin{aligned}
A(z) y(t) & =B(z) \bar{u}(t)+D(z) v(t), \\
A(z) & =1+a_{1} z^{-1}+a_{2} z^{-2}=1-1.10 z^{-1}+0.70 z^{-2}, \\
B(z) & =b_{0}+b_{1} z^{-1}+b_{2} z^{-2}=1+0.55 z^{-1}+0.90 z^{-2}, \\
D(z) & =1+d_{1} z^{-1}=1-0.30 z^{-1}, \\
\bar{u}(t) & =\gamma_{1} u(t)+\gamma_{2} u^{2}(t)=0.45 u(t)+0.60 u^{2}(t), \\
\vartheta & =\left[a_{1}, a_{2}, b_{1}, b_{2}, \gamma_{1}, \gamma_{2}, d_{1}\right]^{\mathrm{T}} \\
& =[-1.10,0.70,0.55,0.90,0.45,0.60,-0.30]^{\mathrm{T}} .
\end{aligned}
$$

In this simulation, the input vector $\{u(t)\}$ is taken as an uncorrelated persistent excitation signal sequence with zero mean and $\{v(t)\}$ as a white noise sequence with zero mean and variances $\sigma^{2}=0.10^{2}$ and $\sigma^{2}=0.50^{2}$. Taking the data length $L=1000$ data, we apply the proposed E-NI algorithm in (17), (18), (19), (20), (21), (22), (23), (24), (25), and (26), the KT-NI algorithm in (39), (40), (41), (42), (43), (44), (45), (46), (47), (48), and (49), and the D-KT-NI algorithm in (62), (63), (64), (65), (66), (67), (68), (69), (70), (71), and (72) to estimate the parameters of the example system; the parameter estimates of each 
TABLE 2: The parameter estimates and errors of the KT-NI algorithm.

\begin{tabular}{cccccccccc}
\hline$\sigma^{2}$ & $k$ & $a_{1}$ & $a_{2}$ & $b_{1}$ & $b_{2}$ & $\gamma_{1}$ & $\gamma_{2}$ & $d_{1}$ \\
\hline & 1 & -1.39971 & 0.79168 & -0.00123 & -0.00620 & 0.41797 & 0.80328 & -0.00689 & 62.45484 \\
& 2 & -1.11611 & 0.71412 & 0.41512 & 0.72162 & 0.45094 & 0.56978 & -0.01914 & 19.38946 \\
& 3 & -1.10182 & 0.69537 & 0.57541 & 0.93598 & 0.45429 & 0.60462 & -0.07944 & 12.09068 \\
$0.10^{2}$ & 4 & -1.09800 & 0.70092 & 0.54331 & 0.88549 & 0.45409 & 0.59402 & -0.26185 & 2.25872 \\
& 5 & -1.09991 & 0.69509 & 0.55565 & 0.90284 & 0.45388 & 0.59980 & -0.29063 & 0.69445 \\
& 10 & -1.10056 & 0.69884 & 0.54936 & 0.89586 & 0.45383 & 0.59723 & -0.30489 & 0.43470 \\
& 15 & -1.10040 & 0.69837 & 0.55037 & 0.89703 & 0.45382 & 0.59763 & -0.30442 & 0.38602 \\
& 20 & -1.10043 & 0.69844 & 0.55022 & 0.89685 & 0.45382 & 0.59757 & -0.30453 & 0.39353 \\
\hline & 1 & -1.35007 & 0.75506 & -0.01352 & -0.00620 & 0.43528 & 0.80979 & -0.00969 & 62.02069 \\
& 2 & -1.11307 & 0.71565 & 0.40087 & 0.75740 & 0.46839 & 0.56276 & -0.13437 & 14.43024 \\
& 3 & -1.10170 & 0.68286 & 0.58704 & 0.95127 & 0.47074 & 0.60626 & -0.26416 & 4.17881 \\
$0.50^{2}$ & 4 & -1.10217 & 0.70483 & 0.52615 & 0.85580 & 0.46942 & 0.57888 & -0.30066 & 3.12057 \\
& 5 & -1.10021 & 0.68548 & 0.56763 & 0.90326 & 0.46933 & 0.59731 & -0.29752 & 1.62874 \\
& 10 & -1.10403 & 0.69630 & 0.54549 & 0.87854 & 0.46914 & 0.58844 & -0.30854 & 1.76861 \\
& 15 & -1.10348 & 0.69460 & 0.54940 & 0.88304 & 0.46914 & 0.59008 & -0.30751 & 1.56660 \\
& 20 & -1.10359 & 0.69491 & 0.54869 & 0.88222 & 0.46914 & 0.58978 & -0.30772 & 1.59993 \\
\hline True values & -1.10000 & 0.70000 & 0.55000 & 0.90000 & 0.45000 & 0.60000 & -0.30000 &
\end{tabular}

TABLE 3: The parameter estimates and errors of the D-KT-NI algorithm.

\begin{tabular}{cccccccccc}
\hline$\sigma^{2}$ & $k$ & $a_{1}$ & $a_{2}$ & $b_{1}$ & $b_{2}$ & $\gamma_{1}$ & $\gamma_{2}$ & $d_{1}$ \\
\hline & 1 & -1.23155 & 0.79509 & 0.52286 & 0.89348 & 0.41594 & 0.56889 & -0.00553 & 16.11602 \\
& 2 & -1.03367 & 0.65908 & 0.54663 & 0.90391 & 0.47140 & 0.62599 & -0.26604 & 4.33402 \\
& 3 & -1.15708 & 0.72762 & 0.52849 & 0.89594 & 0.43727 & 0.57773 & -0.34233 & 3.94531 \\
$0.10^{2}$ & 4 & -1.05106 & 0.67958 & 0.56540 & 0.91451 & 0.46594 & 0.62016 & -0.27119 & 3.26187 \\
& 5 & -1.13731 & 0.71586 & 0.53839 & 0.89625 & 0.44084 & 0.58372 & -0.24959 & 3.23833 \\
& 10 & -1.08228 & 0.69163 & 0.55344 & 0.90582 & 0.45739 & 0.60709 & -0.28489 & 1.30713 \\
& 15 & -1.10542 & 0.70184 & 0.54666 & 0.90170 & 0.45054 & 0.59746 & -0.29649 & 0.38449 \\
& 20 & -1.09554 & 0.69756 & 0.54952 & 0.90347 & 0.45350 & 0.60164 & -0.29610 & 0.39095 \\
\hline & 1 & -1.18817 & 0.76494 & 0.54458 & 0.93310 & 0.42779 & 0.57102 & 0.01268 & 15.85345 \\
& 2 & -1.00303 & 0.63606 & 0.57278 & 0.94755 & 0.47960 & 0.61914 & -0.23259 & 7.02860 \\
& 3 & -1.13160 & 0.70802 & 0.53521 & 0.91149 & 0.45152 & 0.58110 & -0.33064 & 2.46460 \\
$0.50^{2}$ & 4 & -1.05647 & 0.67685 & 0.55353 & 0.91567 & 0.47439 & 0.61744 & -0.27791 & 3.02136 \\
& 5 & -1.12083 & 0.70421 & 0.53813 & 0.90975 & 0.45345 & 0.58761 & -0.33396 & 2.11972 \\
& 10 & -1.08063 & 0.68732 & 0.54608 & 0.91189 & 0.46725 & 0.60713 & -0.30157 & 1.52877 \\
& 15 & -1.09787 & 0.69440 & 0.54221 & 0.91065 & 0.46138 & 0.59886 & -0.31698 & 1.18714 \\
& 20 & -1.09061 & 0.69140 & 0.54374 & 0.91111 & 0.46387 & 0.60236 & -0.31068 & 1.19411 \\
\hline True values & -1.10000 & 0.70000 & 0.55000 & 0.90000 & 0.45000 & 0.60000 & -0.30000 & \\
\hline
\end{tabular}

algorithm and their errors are shown in Tables $1-3$, and their parameter estimation errors $\delta:=\|\widehat{\theta}(t)-\theta\| /\|\theta\|$ and $\delta:=\|\widehat{\vartheta}(t)-\vartheta\| /\|\vartheta\|$ versus $t$ of each algorithm are illustrated in Figures 1-3.

From the simulation results in Tables $1-3$ and Figures 1-3, we can conclude following markers.

(i) For all three algorithms, the parameter estimation errors are getting smaller (in general) as the iterative steps $k$ increases (ii) Both algorithms can produce highly accurate parameter estimates under different noise variances

(iii) When the sizes of Hessian matrices $\left[\widehat{\boldsymbol{\Phi}}_{k}^{\mathrm{T}}(L) \widehat{\boldsymbol{\Phi}}_{k}(L)\right]$ and $\left[\widehat{\boldsymbol{\Psi}}_{k}^{\mathrm{T}}(L) \widehat{\boldsymbol{\Psi}}_{k}(L)\right]$ in E-NI algorithm and KT-NI algorithm expand, two algorithms cost massive computational loads. While using the decomposition technique in the D-KT-NI algorithm, the dimensions of two matrices are trimmed from $\left(n_{a}+n_{b}+m+n_{d}+1\right) \times\left(n_{a}+n_{b}+m+n_{d}+1\right)$ and 


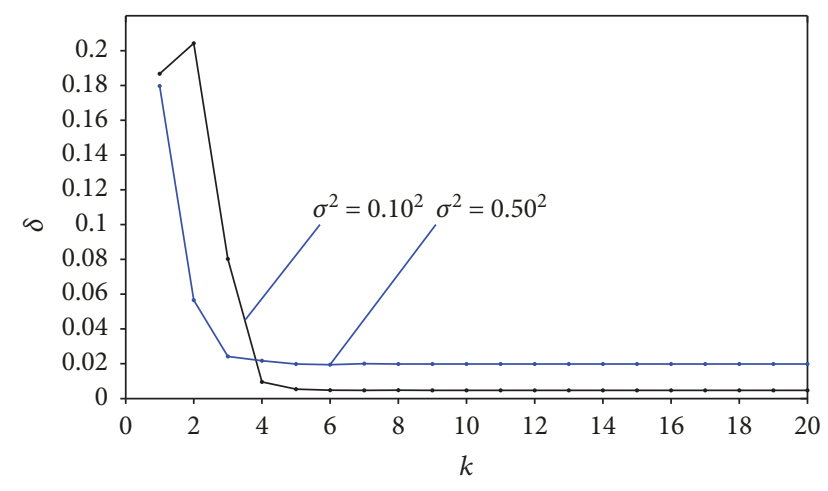

Figure 1: The parameter estimation errors $\delta$ versus $k$ (E-NI).

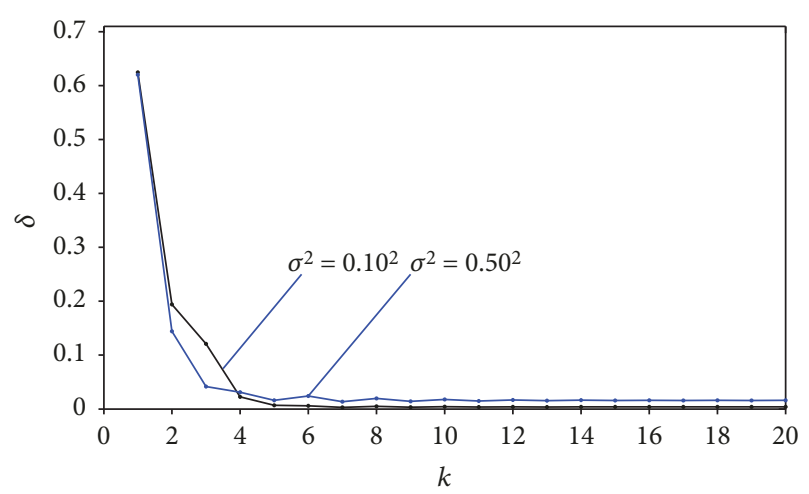

Figure 2: The parameter estimation errors $\delta$ versus $k$ (KT-NI).

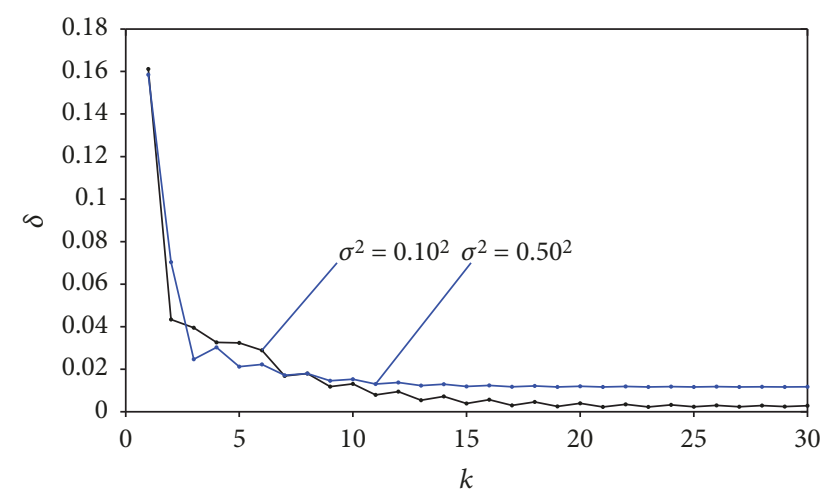

Figure 3: The parameter estimation errors $\delta$ versus $k$ (D-KT-NI).

$\left(n_{a}+n_{b}+m+n_{d}\right) \times\left(n_{a}+n_{b}+m+n_{d}\right) \quad$ to $\quad\left(n_{a}+\right.$ $\left.n_{b}+1\right) \times\left(n_{a}+n_{b}+1\right)$ and $\left(m+n_{d}\right) \times\left(m+n_{d}\right)$. The slimmer Hessian matrices bring better computational efficiency, which is favorable for certain real-time computational situation

\section{Conclusions}

In this work, we have presented extended Newton iterative algorithm, a key term separation-based extended Newton iterative algorithm, and a decomposition-based extended Newton iterative algorithm using the key term separation principle for a class of input nonlinear systems. The illustrative example shows that the decomposition-based extended Newton iterative algorithm using the key term separation principle can produce the high accurate estimates at a relatively lower computational expense. The proposed methods can be further extended to engineering systems [40-42] or other nonlinear scalar or multivariable systems [43-45].

\section{Data Availability}

The data used to support the findings of this study are available from the corresponding author upon request.

\section{Conflicts of Interest}

The authors declare no conflict of interest.

\section{Acknowledgments}

The author is grateful to Professor Feng Ding at the Jiangnan University for his helpful suggestions, and the main idea of this work comes from him and his books. This research was supported by the joint funds of the National Natural Science Foundation of China (Grant No. U1734210), the National Science Foundation for Young Scientists of China (Grant No. 61603156), and the Government of Jiangsu Province's Prospective Research Project (Grant No. BY2015019-29).

\section{References}

[1] D. Zhu, X. Cao, B. Sun, and C. Luo, "Biologically inspired selforganizing map applied to task assignment and path planning of an AUV system," IEEE Transactions on Cognitive and Developmental Systems, vol. 10, no. 2, pp. 304-313, 2018.

[2] J. Na, M. N. Mahyuddin, G. Herrmann, X. Ren, and P. Barber, "Robust adaptive finite-time parameter estimation and control for robotic systems," International Journal of Robust and Nonlinear Control, vol. 25, no. 16, pp. 3045-3071, 2015.

[3] Y. Cao, P. Li, and Y. Zhang, "Parallel processing algorithm for railway signal fault diagnosis data based on cloud computing," Future Generation Computer Systems, vol. 88, pp. 279-283, 2018.

[4] Y. Zhang, Y. Cao, Y. Wen, L. Liang, and F. Zou, "Optimization of information interaction protocols in cooperative vehicleinfrastructure systems," Chinese Journal of Electronics, vol. 27, no. 2, pp. 439-444, 2018.

[5] M. Gan, Han-Xiong Li, and Hui Peng, "A variable projection approach for efficient estimation of RBF-ARX model," IEEE Transactions on Cybernetics, vol. 45, no. 3, pp. 462-471, 2015.

[6] L. Pazzi and M. Pellicciari, "From the Internet of Things to cyber-physical systems: the holonic perspective," Procedia Manufacturing, vol. 11, pp. 989-995, 2017.

[7] C. Yu and M. Verhaegen, "Blind multivariable ARMA subspace identification," Automatica, vol. 66, pp. 3-14, 2016.

[8] C. Yu and M. Verhaegen, "Subspace identification of distributed clusters of homogeneous systems," IEEE Transactions on Automatic Control, vol. 62, no. 1, pp. 463-468, 2017.

[9] J. Na, G. Herrmann, and K. Zhang, "Improving transient performance of adaptive control via a modified reference model and novel adaptation," International Journal of Robust and Nonlinear Control, vol. 27, no. 8, pp. 1351-1372, 2017. 
[10] J. Ding, "Recursive and iterative least squares parameter estimation algorithms for multiple-input-output-error systems with autoregressive noise," Circuits, Systems, and Signal Processing, vol. 37, no. 5, pp. 1884-1906, 2018.

[11] J. Na, J. Yang, X. Wu, and Y. Guo, "Robust adaptive parameter estimation of sinusoidal signals," Automatica, vol. 53, pp. 376384, 2015.

[12] J. Na, J. Yang, X. Ren, and Y. Guo, "Robust adaptive estimation of nonlinear system with time-varying parameters," International Journal of Adaptive Control and Signal Processing, vol. 29, no. 8, pp. 1055-1072, 2015.

[13] C. Wang and T. Tang, "Recursive least squares estimation algorithm applied to a class of linear-in-parameters output error moving average systems," Applied Mathematics Letters, vol. 29, pp. 36-41, 2014.

[14] X. Zhang, F. Ding, F. E. Alsaadi, and T. Hayat, "Recursive parameter identification of the dynamical models for bilinear state space systems," Nonlinear Dynamics, vol. 89, no. 4, pp. 2415-2429, 2017.

[15] Y. Wang and F. Ding, "Recursive least squares algorithm and gradient algorithm for Hammerstein-Wiener systems using the data filtering," Nonlinear Dynamics, vol. 84, no. 2, pp. 1045-1053, 2016.

[16] J. Pan, H. Ma, X. Jiang, W. Ding, and F. Ding, "Adaptive gradient-based iterative algorithm for multivariable controlled autoregressive moving average systems using the data filtering technique," Complexity, vol. 2018, Article ID 9598307, 11 pages, 2018.

[17] J. Ding, "The hierarchical iterative identification algorithm for multi-input-output-error systems with autoregressive noise," Complexity, vol. 2017, Article ID 5292894, 11 pages, 2017.

[18] C. Yang, J. Na, G. Li, Y. Li, and J. Zhong, "Neural network for complex systems: theory and applications," Complexity, vol. 2018, Article ID 3141805, 2 pages, 2018.

[19] J. Li and X. Li, "Particle swarm optimization iterative identification algorithm and gradient iterative identification algorithm for Wiener systems with colored noise," Complexity, vol. 2018, Article ID 7353171, 8 pages, 2018.

[20] Y. Ji and F. Ding, "Multiperiodicity and exponential attractivity of neural networks with mixed delays," Circuits, Systems, and Signal Processing, vol. 36, no. 6, pp. 25582573, 2017.

[21] L. Xu, L. Chen, and W. Xiong, "Parameter estimation and controller design for dynamic systems from the step responses based on the Newton iteration," Nonlinear Dynamics, vol. 79, no. 3, pp. 2155-2163, 2015.

[22] K. Deng and F. Ding, "Newton iterative identification method for an input nonlinear finite impulse response system with moving average noise using the key variables separation technique," Nonlinear Dynamics, vol. 76, no. 2, pp. 1195-1202, 2014.

[23] F. Ding, K. Deng, and X. Liu, "Decomposition based Newton iterative identification method for a Hammerstein nonlinear FIR system with ARMA noise," Circuits, Systems, and Signal Processing, vol. 33, no. 9, pp. 2881-2893, 2014.

[24] F. Ding, H. Chen, L. Xu, J. Dai, Q. Li, and T. Hayat, "A hierarchical least squares identification algorithm for Hammerstein nonlinear systems using the key term separation," Journal of the Franklin Institute, vol. 355, no. 8, pp. 37373752, 2018.
[25] P. Ma, F. Ding, and Q. Zhu, "Decomposition-based recursive least squares identification methods for multivariate pseudo-linear systems using the multi-innovation," International Journal of Systems Science, vol. 49, no. 5, pp. 920-928, 2018.

[26] L. Ma and X. Liu, "Recursive maximum likelihood method for the identification of Hammerstein ARMAX system," Applied Mathematical Modelling, vol. 40, no. 13-14, pp. 6523-6535, 2016.

[27] Y. Mao and F. Ding, "A novel parameter separation based identification algorithm for Hammerstein systems," Applied Mathematics Letters, vol. 60, pp. 21-27, 2016.

[28] J. Vörös, "Parameter identification of Wiener systems with multisegment piecewise-linear nonlinearities," Systems \& Control Letters, vol. 56, no. 2, pp. 99-105, 2007.

[29] J. Li, W. X. Zheng, J. Gu, and L. Hua, "A recursive identification algorithm for Wiener nonlinear systems with linear state-space subsystem," Circuits, Systems, and Signal Processing, vol. 37, no. 6, pp. 2374-2393, 2018.

[30] D. Q. Wang, Z. Zhang, and J. Y. Yuan, "Maximum likelihood estimation method for dual-rate Hammerstein systems," International Journal of Control, Automation and Systems, vol. 15, no. 2, pp. 698-705, 2017.

[31] J. Ma, W. Xiong, J. Chen, and D. Feng, "Hierarchical identification for multivariate Hammerstein systems by using the modified Kalman filter," IET Control Theory \& Applications, vol. 11, no. 6, pp. 857-869, 2017.

[32] Y. Liu and E. W. Bai, "Iterative identification of Hammerstein systems," Automatica, vol. 43, no. 2, pp. 346-354, 2007.

[33] F. Ding, Y. Wang, J. Dai, Q. Li, and Q. Chen, “A recursive least squares parameter estimation algorithm for output nonlinear autoregressive systems using the input-output data filtering," Journal of the Franklin Institute, vol. 354, no. 15, pp. 69386955, 2017.

[34] J. Ma, B. Huang, and F. Ding, "Iterative identification of Hammerstein parameter varying systems with parameter uncertainties based on the variational Bayesian approach," IEEE Transactions on Systems, Man, and Cybernetics: Systems, vol. PP, no. 99, pp. 1-11, 2017.

[35] F. Ding, X. P. Liu, and G. Liu, "Identification methods for Hammerstein nonlinear systems," Digital Signal Processing, vol. 21, no. 2, pp. 215-238, 2011.

[36] L. Xu, "The damping iterative parameter identification method for dynamical systems based on the sine signal measurement," Signal Processing, vol. 120, pp. 660-667, 2016.

[37] L. Xu, "The parameter estimation algorithms based on the dynamical response measurement data," Advances in Mechanical Engineering, vol. 9, no. 11, pp. 1-12, 2017.

[38] H. Chen, Y. Xiao, and F. Ding, "Hierarchical gradient parameter estimation algorithm for Hammerstein nonlinear systems using the key term separation principle," Applied Mathematics and Computation, vol. 247, pp. 1202-1210, 2014.

[39] J. Vörös, "Identification of nonlinear cascade systems with output hysteresis based on the key term separation principle," Applied Mathematical Modelling, vol. 39, no. 18, pp. 55315539, 2015.

[40] X. Li and D. Q. Zhu, "An improved SOM neural network method to adaptive leader-follower formation control of AUVs," IEEE Transactions on Industrial Electronics, vol. 65, no. 10 , pp. 8260-8270, 2018. 
[41] Y. Cao, L. Ma, S. Xiao, X. Zhang, and W. Xu, "Standard analysis for transfer delay in CTCS-3," Chinese Journal of Electronics, vol. 26, no. 5, pp. 1057-1063, 2017.

[42] Y. Cao, Y. Wen, X. Meng, and W. Xu, "Performance evaluation with improved receiver design for asynchronous coordinated multipoint transmissions," Chinese Journal of Electronics, vol. 25, no. 2, pp. 372-378, 2016.

[43] X.-F. Li, Y.-D. Chu, A. Y. T. Leung, and H. Zhang, "Synchronization of uncertain chaotic systems via complete-adaptiveimpulsive controls," Chaos, Solitons \& Fractals, vol. 100, pp. 24-30, 2017.

[44] J. Pan, X. Jiang, X. Wan, and W. Ding, “A filtering based multiinnovation extended stochastic gradient algorithm for multivariable control systems," International Journal of Control, Automation and Systems, vol. 15, no. 3, pp. 1189-1197, 2017.

[45] L. Xu and F. Ding, "Parameter estimation for control systems based on impulse responses," International Journal of Control, Automation and Systems, vol. 15, no. 6, pp. 2471-2479, 2017. 


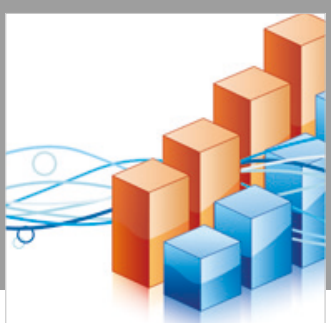

Advances in

Operations Research

\section{-n-m}
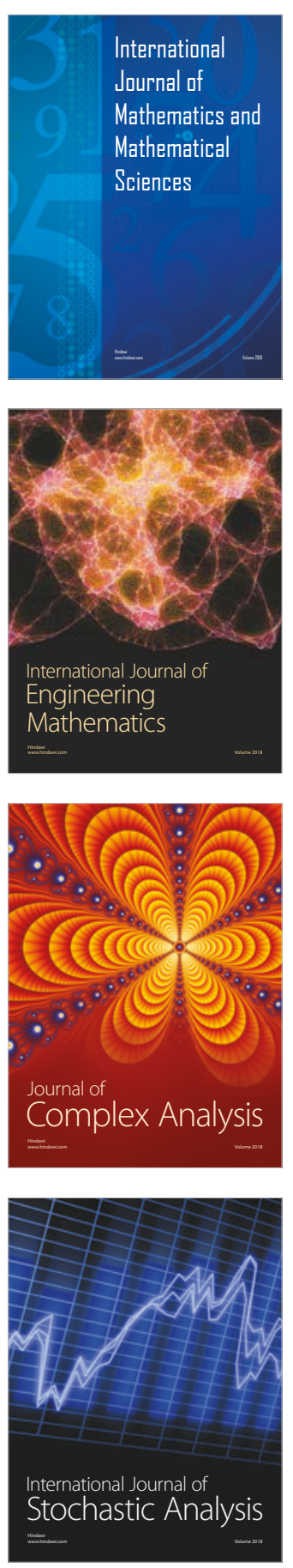
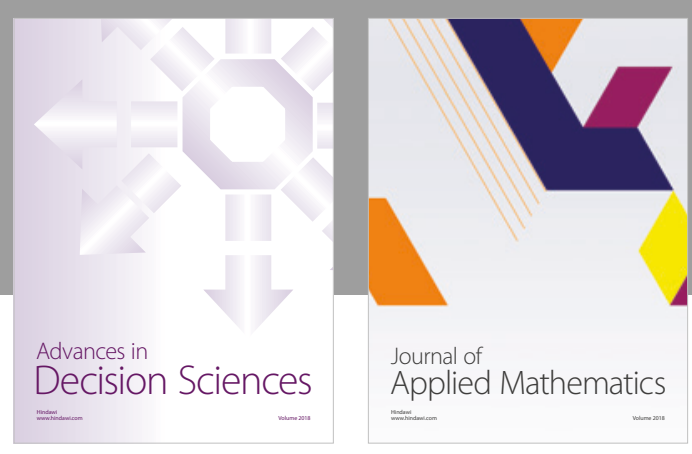

Journal of

Applied Mathematics
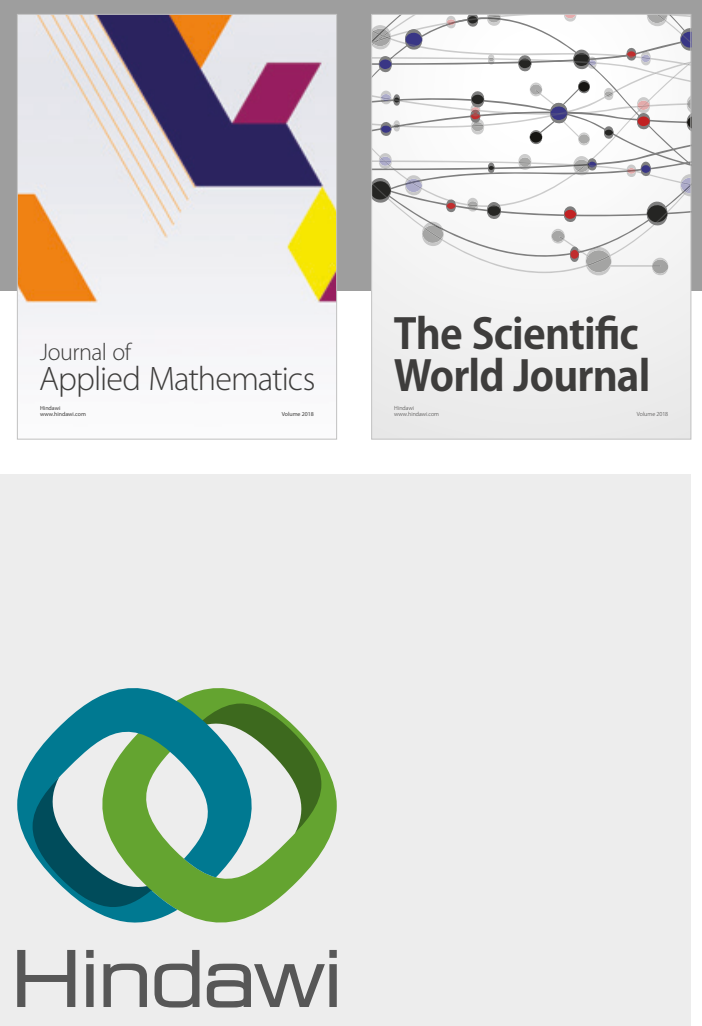

Submit your manuscripts at

www.hindawi.com

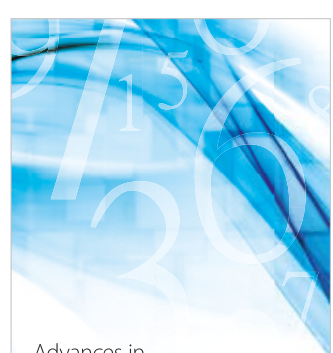

Advances in
Numerical Analysis
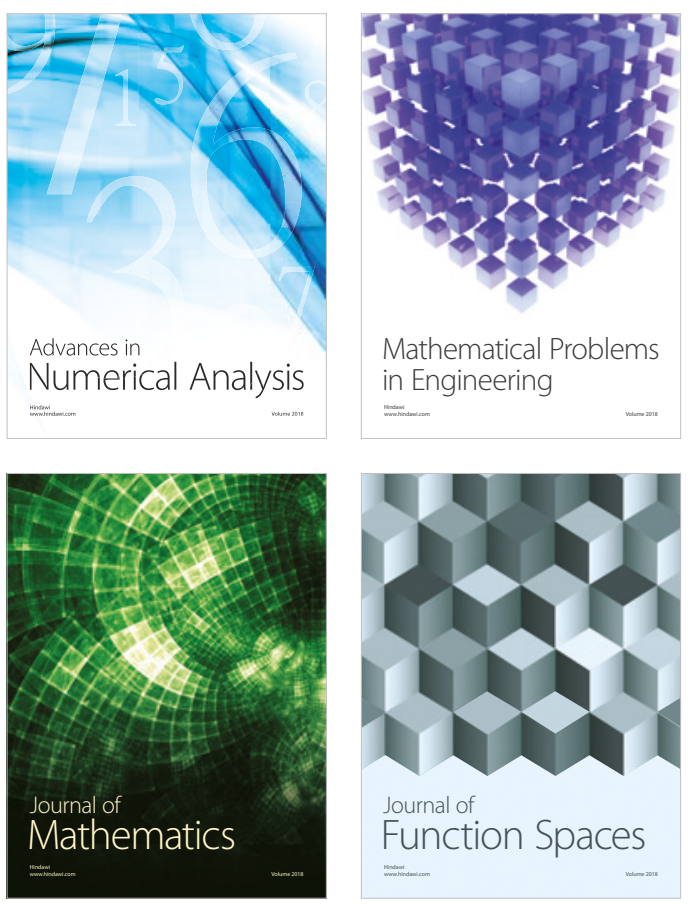

Mathematical Problems in Engineering

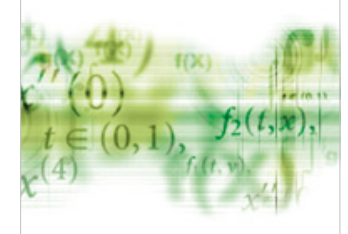

International Journal of

Differential Equations

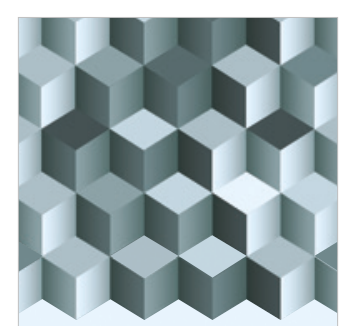

Journal of

Function Spaces
The Scientific

World Journal

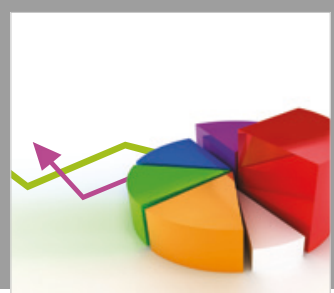

Journal of

Probability and Statistics
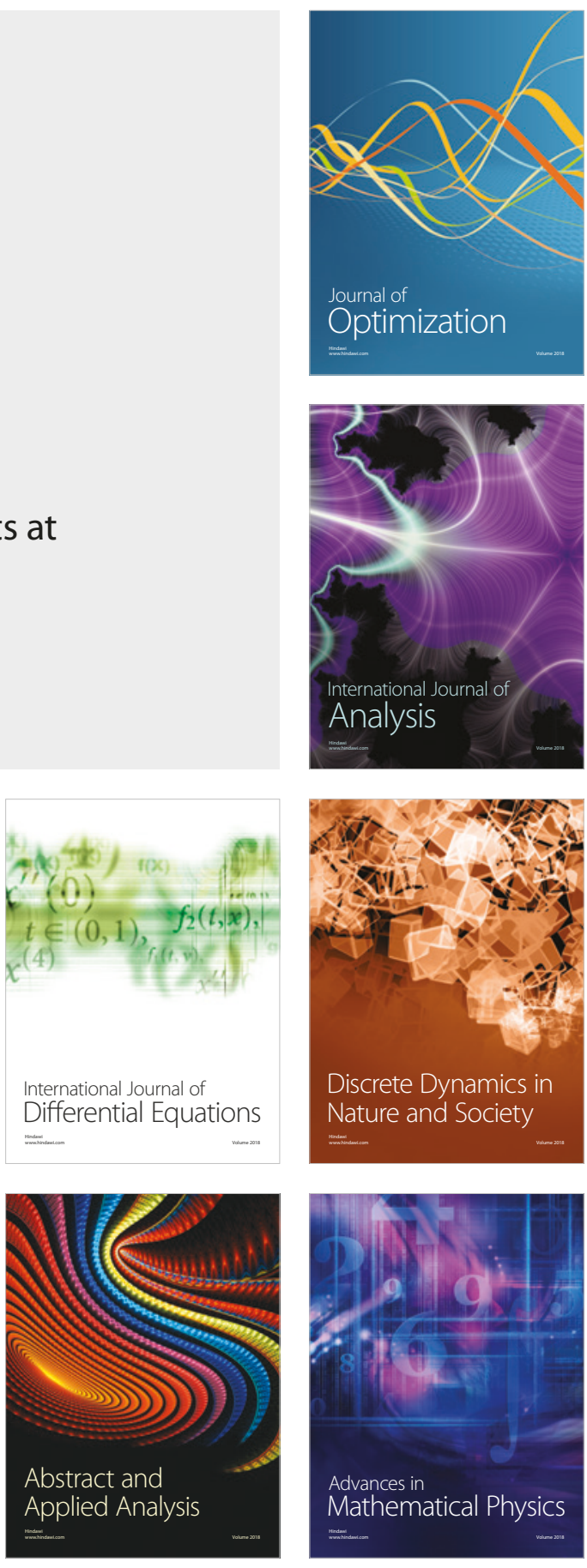